\title{
Transverse and Longitudinal proximity effect
}

\author{
Priyanka Jalan ${ }^{1 *}$, Hum Chand ${ }^{1}$, Raghunathan Srianand ${ }^{2}$ \\ ${ }^{1}$ Aryabhatta Research Institute of observational sciencES (ARIES), Nainital 263002, India \\ ${ }^{2}$ Inter-University Centre for Astronomy and Astrophysics (IUCAA), Post Bag 4, \\ Pune 411007, India
}

\begin{abstract}
With close pairs ( $\leq 1.5 \operatorname{arcmin})$ of quasars (QSOs), absorption in the spectra of a background quasar in the vicinity of a foreground quasar can be used to study the environment of the latter quasar at kpc-Mpc scales. For this we used a sample of 205 quasar pairs from the Sloan Digital Sky-Survey Data Release 12 (SDSS DR12) in the redshift range of 2.5 to 3.5 by studying their $\mathrm{H}$ I Ly- $\alpha$ absorption. We study the environment of QSOs both in the longitudinal as well as in the transverse direction by carrying out a statistical comparison of the Ly- $\alpha$ absorption lines in the quasar vicinity to that of the absorption lines caused by the inter-galactic medium (IGM). This comparison was done with IGM, matched in absorption redshift and signal-to-noise ratio (SNR) to that of the proximity region. In contrast to the measurements along the line-of-sight, the regions transverse to the quasars exhibit enhanced H I Ly- $\alpha$ absorption. This discrepancy can either be interpreted as due to an anisotropic emission from the quasars or as a consequence of their finite lifetime.
\end{abstract}

\section{Introduction}

Different types of absorption lines are seen bluewards of the Ly- $\alpha$ emission in the spectra of quasars allowing us to study various properties of the intervening absorbers interpreted as clouds of the primordial material. The bound on the density of the neutral hydrogen, $n_{H}$, by Gunn \& Peterson (1965) requires that the intergalactic medium must be almost ionized, in ionization equilibrium with the meta-galactic ultraviolet (UV) background. Locally, the UV-field may be dominated by the ionization from a single source leading to a deficit of absorption lines sufficiently close to these sources widely known as the 'inverse' or 'proximity'effect. It is also expected that the gas nearby to the QSOs must have a higher than average density compared to that of the intergalactic medium (IGM) because QSOs reside in massive galaxies with large halo masses. Therefore, in the environment of the QSOs, there exists a degeneracy between the ionisation from the QSOs (decreasing the optical depth) and excess of overdensity (increasing the optical depth) in which the QSOs seem to reside. Therefore, in order to study the transverse proximity effect (TPE) we need closely separated pairs and a control sample of IGM for the comparison. This requirement is fulfilled, thanks to the SDSS DR12 quasar catalog which contains spectral information for 297,301 quasars. We need closely separated QSO pairs, as the background (b/g) spectra are used to see the effect of the foreground (f/g) QSOs in the transverse direction while the spectra of the f/g QSOs themselves serve the purpose for the analysis of the longitudinal proximity effect (LPE). In this paper, we study TPE and LPE (i) by using a sample of f/g QSOs of the 205 QSO pairs (SDSS DR12) for ionisations of both the LPE and TPE regions, (ii) use the control sample of IGM matching both in the SNR as well as in the redshift to those of the proximity regions (e.g., see section 2) and (iii) use pixel-optical depth statistics to independently confirm or refute the excess of such an overdensity around the QSOs (e.g., see section 3). Finally, we study the

*email: priyanka@aries.res.in 
average overdensity profile around the QSOs, after the ionisation scaling and the possibilities of the discrepancy in TPE and LPE (e.g., see section 4).

\section{Data and sampling}

We have used the spectroscopic databases of the SDSS DR12 quasar catalog. Using this catalog we have selected our sample by imposing the following selection criteria (i) $z_{e m}>2.5$, resulting in 83,661 QSOs so that the Ly- $\alpha$ forest can be observed in the SDSS wavelength range, (ii) pair separation $\theta<1.5^{\prime}$, to probe the environment on kpc scales resulting in 1,344 pairs, (iii) the $z_{e m}^{b g}-z_{e m}^{f g}<0.5$, so that the Ly- $\alpha$ emission line of the $\mathrm{f} / \mathrm{g}$ QSOs is present in between the Ly- $\beta$ and Ly- $\alpha$ emission lines of the b/g QSOs leading to only 380 pairs out of 1,344 pairs, (iv) Broad Absorption Lines (BAL) QSOs are excluded to avoid any extra ionisation caused by the outflows, which removed another 110 pairs, (v) no DLA should be present in the proximity region which would lead to an overdensity, leading to 260 pairs, (vi) excluding the QSOs (29 pairs) which do not have a well-defined redshift even after adopting the algorithm of Hewett et al. (2010) to improve the redshift of our sample and (vii) the velocity difference between the f/g and the b/g QSOs should be greater than $2000 \mathrm{~km} \mathrm{~s}^{-1}$, to avoid binary QSOs, which excluded another 26 pairs. We ended with a sample of 205 closely separated pairs, with a maximum transverse separation of $700 \mathrm{kpc}$ at $z \sim 3$.

\subsection{Control sample of the IGM Ly- $\alpha$ forest matching in redshift and SNR with that of the proximity region}

For optical depth statistics based on the pixel by pixel optical depth method which is used here, it is important to remove the strong redshift evolution effect of the optical depths while combining the optical depths from the Ly- $\alpha$ absorption at different redshifts. One possibility is to scale the optical depth to one reference value say $z_{r e f}$ for both the IGM as well as the proximity region as used by Rollinde et al. (2005). However, given the fact that the observed optical depth distribution has upper and lower limits based on the noise in the continuum and in the core of the saturated absorption lines, as a result, redshift scaling may artificially introduce the optical depths beyond these ranges and may bias the statistics by creating an artificial difference in two distributions viz. the IGM and the proximity region. Therefore, we used a control sample of IGM for the TPE study i.e, we chose another random sightline of non-BAL QSOs from the SDSS DR12 with Ly- $\alpha$ forest such that (i) it matches with that of the proximity region in continuum SNR (within \pm 1 ) and in the Ly- $\alpha$ forest absorption redshift range, (ii) $z_{e m}$ is such that all the pixels used for the control sample of the IGM are not under the influence of its own QSO ionisation (i.e, $\mathrm{r}_{\|}>15 \mathrm{Mpc}$ from $z_{I G M}^{q s o}$ ) and (iii) the sightline is at $\mathrm{r}_{\perp}>15 \mathrm{Mpc}$ from the f/g QSO $\left(\right.$ at $z_{f g}$ ) of the pair so as to be free from any proximity of the f/g QSO of the pair. A similar procedure was also adopted to make the control sample of IGM for the longitudinal proximity study. As evident from Fig.1, the IGM control sample is in a very good agreement with that of the proximity range in redshift and the continuum SNR. We used this IGM control sample for the further study of TPE and LPE.

\section{Analysis}

The transmitted flux of a quasar spectrum at $\mathrm{i}^{\text {th }}$ pixel is given by $F^{\prime}\left(\lambda_{i}\right)=F_{\text {cont }}\left(\lambda_{i}\right) e^{-\tau\left(\lambda_{i}\right)}$, where $F_{\text {cont }}\left(\lambda_{i}\right)$ is the continuum and $\tau\left(\lambda_{i}\right)$ is the optical depth due to an absorbing cloud at the $\mathrm{i}^{\mathrm{th}}$ pixel. We have computed the cumulative probability distribution function (CPDF) of the pixel optical depth values to study any significant difference between these two regions with and without the proximity effect matched in redshift and SNR. We discarded here the optical depth (i.e, $\tau_{i}=-\log \left(F^{\prime}\left(\lambda_{i}\right) / F_{\text {cont }}\left(\lambda_{i}\right)\right)$ ) values outside the range of $\tau_{\min }=$ $-\log (1-3 \sigma) \simeq 0.01$ and $\tau_{\max }=-\log (3 \sigma) \simeq 2.35$, where $\sigma$ is the typical rms noise of the normalised flux in the unabsorbed spectral region of our sample. These lower and upper constraints permit to avoid the contaminations of the CPDF by optical depths from the continuum and the saturated region pixels, respectively. We show in Fig. 2 (left panel), the CPDF of the optical depth in the transverse region as compared to that of the IGM control sample within a region $r \leq 2 \mathrm{Mpc}$ from the $\mathrm{f} / \mathrm{g}$ QSO. As can be seen from the figure, the 
overdensity is quite dominant in the transverse direction. Similarly, we have plotted in Fig. 2 (right panel) the CPDF for the longitudinal direction which shows that the ionisation from the f/g QSO is evident. From the figure it can be concluded that excess overdensity is evident in the transverse direction as compared to the longitudinal direction. In order to get a clear picture of any dissimilarity between the TPE and LPE regions, we have to account for the ionisation from the f/g QSO in both directions as elaborated in the next section.
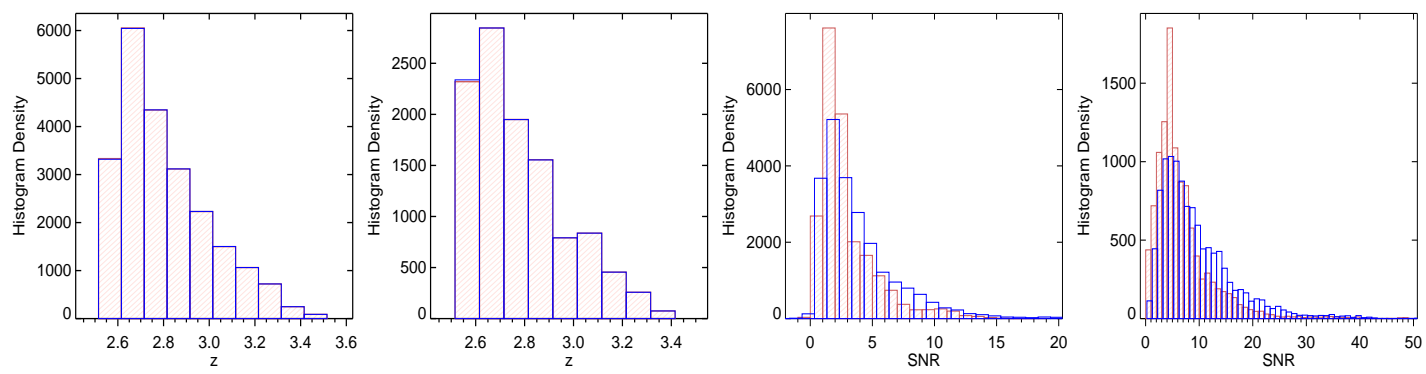

Figure 1: (1.) Redshift matching of the b/g QSO (red: shaded) with QSO used for IGM (blue: line). (2.) Same as (1) but for LPE (f/g). (3.) SNR matching of TPE region (red: shaded) with IGM (blue: line). (4.) Same as (3) but for LPE.

\subsection{Ionisation and overdensity in the proximity region}

An absorbing cloud in the proximity region at a distance $r$ from the $\mathrm{f} / \mathrm{g}$ QSO will be in photoionisation equilibrium with the QSO, with photoionisation rate, $\Gamma_{Q S O}(r)=\int_{\nu_{912}}^{\infty} \frac{L_{\nu}}{4 \pi r^{2}} \frac{\sigma_{H I}}{h \nu} d \nu$. We have computed the luminosity of the $\mathrm{H}$ I ionising photons due to the f/g QSO (by assuming that the QSOs emit isotropically) as $L_{912}=F_{912} \times 4 \pi d_{L}^{2}$ where the flux is the extinction corrected flux in the observer's rest frame and $d_{L}$ is the luminosity distance. To compute the rest frame flux at $912 \AA$ we first computed the rest frame flux in the line free region around $1695 \AA$ and then extrapolated to $912 \AA$ by using a broken power law having the form, $L_{\nu} \propto \nu^{-0.44}$ for $\lambda>1300 \AA$ and $\propto \nu^{-1.57}$ for $\lambda<1300 \AA$ (e.g., see Khaire et al. 2015). The relative importance of the ionisation from the quasar versus the UV-background ionizing photons in the proximity region will be characterized by $\left(1+\omega_{r}\right) \propto \Gamma_{Q S O}(r) / \Gamma_{I G M}(z)$, where $\Gamma_{I G M}$ is the photoionisation rate by the UV background radiation and is taken from Khaire et al. (2016). As explained by Rollinde et al. (2005), the combined effect of any density enhancement and extra ionizing photons around the QSOs, is to shift the optical depth $\tau_{0}$ by a factor

$$
\tau_{0} \rightarrow \tau_{0} \frac{(\rho(r) / \bar{\rho})^{1 /(1+\beta)}}{1+\omega_{r}}
$$

where $\bar{\rho}$ is the mean density of the IGM. In order to compensate for the extra ionisation by the f/g QSO we have scaled each pixel in the proximity region as $\tau^{i} \times\left(1+\omega_{r}^{i}\right)$. We have plotted the ratio of the weighted mean optical depth of the proximity region to the weighted mean optical depth of the corresponding IGM at various radial distance bins, in Fig. 3, using the optical depths of the proximity region with and without the $\omega_{r}$ scaling. From the left panel of Fig. 3 based on without $\omega_{r}$ scaling, for $r<5 \mathrm{Mpc}$ in the transverse direction is probably overdensity dominated while in the longitudinal direction, ionisation seems to be dominant up to $r<5 \mathrm{Mpc}$. The majority of this difference disappears after applying the $\omega_{r}$ scalings as shown in the right panel of this figure. However, an overdensity profile shows the difference in the magnitude of the overdensity along the longitudinal and the transverse direction. We have quantified this trend by fitting an exponential function of the form

$$
\tau_{\text {prox }} / \tau_{I G M}=a \times \exp (-r / b)+c
$$

where the best-fit parameters i.e, a, $b$ and $\mathrm{c}$ for the cases of TPE and LPE are $1.31 \pm 0.27,1.50 \pm 0.30,1.04 \pm 0.02$ and $5.39 \pm 5.99,0.34 \pm 0.18,0.93 \pm 0.02$, respectively. This larger magnitude of excess overdensity in the transverse direction can be explained by the anisotropic emission from the QSOs or their finite episodic lifetime as explained in the following section. 

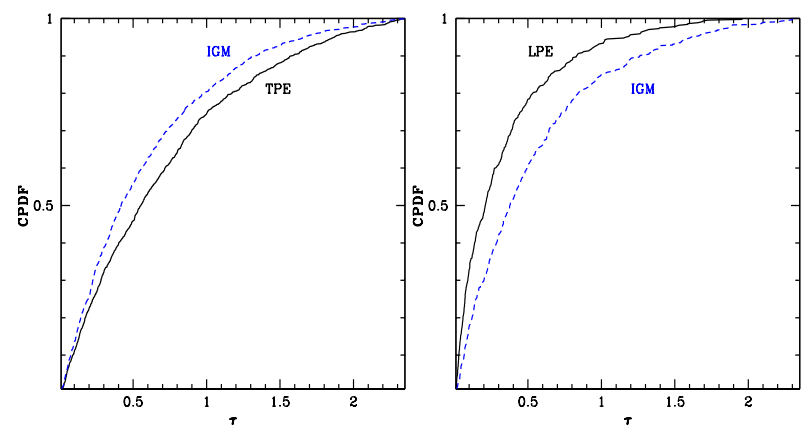

Figure 2: Left: CPDF of TPE (black: solid) region for $\mathrm{r}<2 \mathrm{Mpc}$ versus the redshift and SNR matched IGM (blue: dashed). Right: Same as the left panel but for the LPE region.
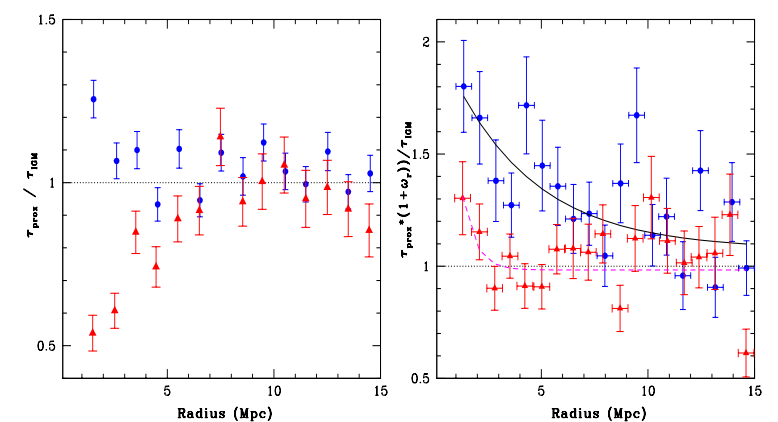

Figure 3: Left: Weighted mean of the optical depth ratio $\left(\tau_{\text {prox }} / \tau_{I G M}\right)$ in a radial bin is plotted for the TPE region (blue: circle) and the LPE region (red: triangle). Right: same as the left but the optical depth of the proximity region is scaled by $1+\omega_{r}$.

\section{Results and discussion}

From our analysis, we conclude that in the transverse direction the overdensity seems to dominate in contrast to that along longitudinal direction where the ionisation from the QSOs overshines the overdensity. Similar results were obtained in the past by Prochaska et al. (2013). Our analysis has shown the difference in the excess overdensity in the longitudinal and the transverse direction at the $95 \%$ confidence level based on KS statistics up to $5 \mathrm{Mpc}$. One possibility to understand this discrepancy can be that the actual f/g QSOs emission might be anisotropic. As a result, the ionisation scaling $\left(1+\omega_{r}\right)$ which we have computed using the luminosity seen in the longitudinal direction might be more than the actual value if the QSOs have beamed emission towards the longitudinal direction. Another possibility of this excess overdensity could be the finite lifetime or perhaps an episodic QSOs phase as also conjectured by Kirkman et al. (2008). In this scenario, the radiation from the $\mathrm{f} / \mathrm{g}$ QSOs will take an extra time $\left(\mathrm{R}_{\perp} / \mathrm{c}\right)$ to reach the gas in the transverse direction. Therefore, if the $\mathrm{f} / \mathrm{g}$ QSO's active phase lifetime is smaller than $\mathrm{R}_{\perp} / \mathrm{c}$, then it is possible that we may not see the $\mathrm{f} / \mathrm{g}$ QSO radiation effect on the gas in the transverse direction (along b/g QSO sightline) when we observe the $\mathrm{f} / \mathrm{g}$ QSOs in its active QSO phase. If this is the correct interpretation, then the QSOs episodic lifetime for the difference in the excess overdensity seen within a $2 \mathrm{Mpc}$ transverse distance bin will correspond to about $6 \mathrm{Myr}$ (i.e, $2 \mathrm{Mpc} / \mathrm{c}$ ). In order to confirm any of these possibilities we need pairs which are at even smaller separations and the spectra of pairs should be of high resolution and high SNR in order to remove any discrepancy because of SNR. If the anisotropy still persists, say at the $1 \mathrm{kpc}$ scale, then we can rule out the possibility of a finite episodic lifetime of the QSOs because from the theoretical evidence we know that the QSOs can not have a very small lifetime. In the future, we will use the $3.6 \mathrm{~m}$ DOT to observe such closely separated pairs using long-slit spectroscopy.

\section{Acknowledgements}

We thank Vikram Khaire for providing us the UV-background photo-ionisation rate at various redshifts.

\section{References}

Gunn J. E., Peterson B. A. 1965, ApJ, 142, 1633

Hewett P.C., Wild V. 2010, MNRAS, 405, 2302

Khaire V., Srianand R. 2015, ApJ, 805, 33

Khaire V., Srianand R., Choudhury T. R., Gaikwad P. 2016, MNRAS, 457, 4051

Kirkman D., Tytler D. 2008, MNRAS, 391, 1457

Prochaska J. X., Hennawi J. F., Lee K.-G. et al. 2013, ApJ, 776, 136

Rollinde E., Srianand R., Theuns T., Petitjean P, Chand H. 2005, MNRAS, 361, 1015 